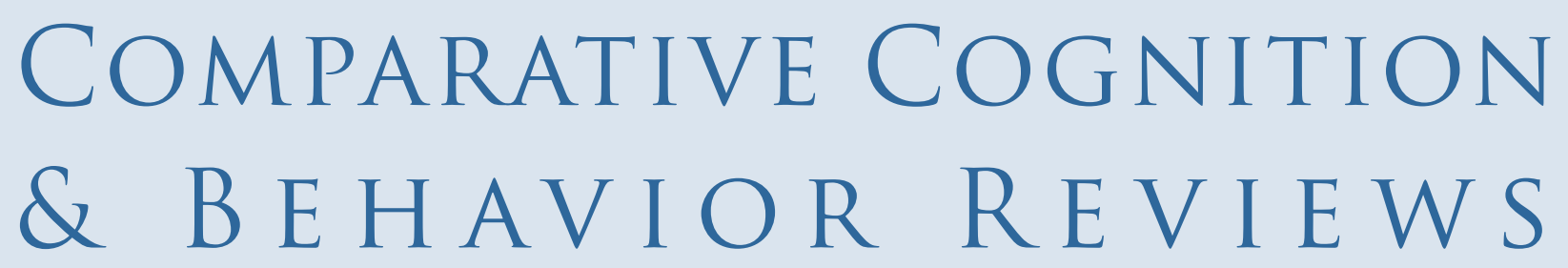

\title{
Environmental Influences on Spatial Memory and the Hippocampus in Food-Caching Chickadees
}

\author{
Vladimir V. Pravosudov \\ Department of Biology, University of Nevada, Reno, USA
}

Lara D. LaDage

Department of Biology, Penn State, Altoona, USA

\author{
Timothy C. Roth II \\ Department of Psychology, Franklin and Marshall College, USA
}

\author{
Cody A. Freas \\ Department of Biological Sciences, \\ Macquarie University, Australia
}

Cognitive abilities have been widely considered as a buffer against environmental harshness and instability, with better cognitive abilities being especially crucial for fitness in harsh and unpredictable environments. Although the brain is considered to be highly plastic and responsive to changes in the environment, the extent of such environment-induced plasticity and the relative contributions of natural selection to the frequently large variation in cognitive abilities and brain morphology both within and between species remain poorly understood. Food-caching chickadees present a good model to tackle these questions because they: (a) occur over a large gradient of environmental harshness largely determined by winter climate severity, (b) depend on food caches to survive winter and their ability to retrieve food caches is, at least in part, reliant on hippocampus-dependent spatial memory, and (c) regularly experience a distinct seasonal cycle of food caching and cache retrieval. Here we review a body of work, both comparative and experimental, on two species of food-caching chickadees and discuss how these data relate to our understanding of how environment-induced plasticity and natural selection generate environment-related variation in spatial memory and the hippocampus, both across populations as well as across seasons within the same population. We argue that available evidence suggests a relatively limited role of environmentinduced structural hippocampal plasticity underlying population variation. At the same time, evidence is consistent with the history of natural selection due to differences in winter climate severity and associated with heritable individual variation in spatial memory and the hippocampus. There appears to be no clear direct association between seasonal variation in hippocampus morphology and seasonal variation in demands of food caching. Finally, we suggest that experimental studies of hippocampal plasticity with captive birds should be viewed with some caution because captivity is associated with large reductions in many hippocampal traits, including volume and in some cases neurogenesis rates, but not neuron number. Comparative studies using captive birds, on the other hand, appear to provide more reliable results, as captivity does not appear to override population differences, especially in the number of hippocampal neurons.

Keywords:

spatial memory; hippocampus; neurogenesis; neurons; plasticity; natural selection; food caching; environment; winter; ambient temperature; seasonality; chickadee 
A key evolutionary question for understanding how environmental heterogeneity is associated with cognitive abilities concerns the relative contribution of environmentinduced effects (e.g., plasticity) and natural selection acting on heritable cognitive traits as a means of generating environment-related variation in cognition and neural traits (e.g., Pravosudov \& Roth, 2013). At least in humans, there is sufficient evidence that both general cognition and specific cognitive traits are highly heritable and that individual variation in these traits is, at least in part, determined by genetics (e.g., Ando, Ono, \& Wright, 2001; Haworth et al., 2010; McGee, 1979; Pedersen, Plomin, Nesselroade, \& McClearn, 1992; Plomin, Pedersen, Lichtenstein, \& McClearn, 1994; Plomin \& Spinath, 2002). Assuming that heritability of cognitive traits is not a unique human phenomenon but is common in other animals, it should provide ample opportunities for natural selection to generate variation in cognitive traits given different selection pressures. Many species occur over a large range of environmental conditions and experience major seasonal changes in their environment. Both geographic and seasonal variation in environmental conditions are likely to impart different demands on cognitive abilities, which may be especially important for fitness in harsher environments (e.g., longer winter period, lower temperatures, more snow cover covering foraging substrates and more frequent snowfalls, etc.) with higher energetic demands (due to lower temperatures) and a shortage of naturally available food (e.g., Pravosudov \& Clayton, 2002; Pravosudov \& Roth, 2013). It is important to note that the range of seasonal variation is usually also associated with geographic variation with a larger range of seasonal variation in harsher environments (e.g., more northern environments are associated with stronger seasonal differences).

Food-caching chickadees present a good case to understand the relationship between the environment, cognition, and the brain because (a) they occur over a large gradient of environmental harshness with different demands on caching and cache retrieval, (b) caching and cache retrieval depend, at least in part, on hippocampus-dependent spatial memory, and (c) they exhibit highly seasonal food caching behavior.

Acknowledgements: Vladimir Pravosudov was supported by NSF awards IOS1351295 and IOS0918268 and Lara LaDage was supported by NSF award IOS0918268. We would like to thank Chris Sturdy and three anonymous reviewers for their constructive criticisms that significantly improved the manuscript.

\section{Population Variation in Spatial Memory and Hippocampus Morphology Is Associated with Differences in Winter Climate Harshness}

Food-caching chickadees occur over a large range of environmental conditions with some populations experiencing relatively milder winters and some others experiencing relatively harsher winters. Chickadees are non-migratory birds that spend the non-breeding season in social groups characterized by linear social dominance hierarchy (e.g., Ekman, 1989; Hogstad, 1989) and appear to rely on food caches to survive winters (e.g., Pravosudov \& Smulders, 2010). Most food-caching chickadee species live in temperate climates where the highest rates of mortality likely occur during the winter, likely due to the inability to meet energetic requirements. During the winter naturally available food is both in short supply and unpredictable in availability. Thus, food caching has been widely hypothesized to have evolved to provide a more reliable food supply during that time (Krebs, Sherry, Healy, Perry, \& Vaccarino, 1989; Pravosudov \& Clayton, 2002; Pravosudov \& Roth, 2013; Sherry, Vaccarino, Vuckenham, \& Herz, 1989; Vander Wall, 1990). At the same time, the large variation in winter harshness associated with climate severity (colder temperatures, more snowfall, longer winter period) across species ranges might be expected to influence the reliance on food caches, depending on winter climate (Pravosudov \& Clayton, 2002; Pravosudov \& Roth, 2013). Longer winter periods means longer periods without abundant and predictable food supply associated with phenology of main natural food sources (e.g., invertebrates). Colder temperature is likely associated with higher food intake requirements, yet during the winter naturally available food is limited and unpredictable, and more snow (covering both ground and frequently tree branches) likely reduces access to already limited food. In food-caching birds, food caches appear to represent the main reliable food source during the winter, and harsher winter conditions can be expected to increase reliance on food caches for overwinter survival.

It is well established that spatial memory plays a role in successful cache retrieval and, potentially, even in generating the optimal density of caches during caching (e.g., Male \& Smulders, 2007), so variation in winter climate harshness could be expected to produce differential demands on spatial memory ability (Pravosudov \& Roth, 2013). Birds living in harsher winter environments should benefit from a superior spatial memory that allows them to be more successful in retrieving previously made caches compared to birds wintering in milder climates (Pravosudov \& Clayton, 2002). As spatial memory is dependent, at least in part,

COMPARATIVE COGNITION \& BEHAVIOR REVIEWS 
on the hippocampus, differences in spatial memory among populations that are due to differential dependence on food caches for survival should also be associated with differences in the hippocampus (Pravosudov \& Roth, 2013). Such expected differences in spatial memory and the hippocampus might come about via environment-induced plastic phenotypic responses associated with the differential use of memory (Clayton, 1996, 2001; Clayton \& Krebs, 1994; Woollett \& Maguire, 2011) and/or could be based on genetic differences produced by natural selection if differences in memory and hippocampus morphology are based on heritable mechanisms (Krebs et al., 1989; Pravosudov \& Roth, 2013; Sherry et al., 1989). Before discussing the origin of potential population differences in spatial memory and the hippocampus, we shall first consider the data demonstrating such population differences.

Our studies focused on two species of food-caching chickadees - the black-capped chickadee (Poecile atricapillus) and the mountain chickadee (P. gambeli). Black-capped chickadees occur over a large range on the North American continent that spans large variation in winter conditions both longitudinally and latitudinally (Figure 1; Pravosudov \& Clayton, 2002). Along the latitudinal gradient of winter climate harshness, the black-capped chickadee range expands from a milder climate in Kansas to a much harsher winter climate in Alaska, whereas along the longitudinal gradient, chickadees range from milder climate in Washington state to much harsher winter climate in Maine (Figure 1). The first study compared chickadees from the two most different populations (from most extremely different winter environments) from Alaska (Anchorage) and Colorado and reported that chickadees from Alaska (harsh winters) had a stronger propensity to cache food, significantly better spatial, but not nonspatial memory ability, larger relative and absolute hippocampus volume, and a significantly larger total number of hippocampal neurons (Pravosudov \& Clayton, 2002). The follow-up studies (Roth, LaDage, \& Pravosudov, 2011; Roth \& Pravosudov, 2009) compared 10 populations of black-capped chickadees along the winter climate gradient, including the two populations previously compared in Pravosudov and Clayton (2002). These studies showed that independent of latitudinal differences in day length (shorter in northern populations), harsher winter climatic conditions were associated with larger hippocampus volume, higher total number and larger soma size of hippocampal neurons, larger total number of hippocampal glial cells, and higher neurogenesis rates (Figure 2; Chancellor, Roth, LaDage, \& Pravosudov, 2011; Freas, Bingman, LaDage, \& Pravosudov, 2013; Roth et al., 2011; Roth \& Pravosudov, 2009).
Mountain chickadees experience different winter conditions on a much smaller spatial scale along an elevation gradient of winter climate severity in the mountains, with birds at higher elevations experiencing longer and colder winters (Freas, LaDage, Roth, \& Pravosudov, 2012). Higher elevations are associated with significantly lower winter temperatures (likely requiring more food intake to meet higher energetic demands), longer winter period associated with limited natural (e.g., not cached) food supply (likely increasing reliance on food caches for overwinter survival), and significantly more snow cover (both on the ground and on trees) that likely limits access to some potential foraging substrates. Similarly to black-capped chickadees from different winter conditions, mountain chickadees from higher elevations in the Sierra Nevada had a stronger propensity to cache food, better spatial memory ability, larger hippocampus volume, higher total number and larger soma size of hippocampal neurons, and higher hippocampal neurogenesis rates (Figure 3; Freas et al., 2012; Freas, Bingman, et al., 2013; Freas, Roth, LaDage, \& Pravosudov, 2013).

Overall, these combined data on 10 populations of black-capped chickadees (with the data on two of these populations collected twice during different years) and

Figure 1. Sampling locations across winter climate severity gradients in black-capped chickadees. AKF_-Alaska, Fairbanks; AKA-Alaska, Anchorage; $\mathrm{BC}$-British Columbia; WA-Washington State; MT_Montana; MN_Minnesota; ME_Maine; CO_Colorado;

KS - Kansas; IA—lowa. L-large hippocampus, S-small hippocampus, S-I_small-intermediate hippocampus. Based on Pravosudov et al. (2012).

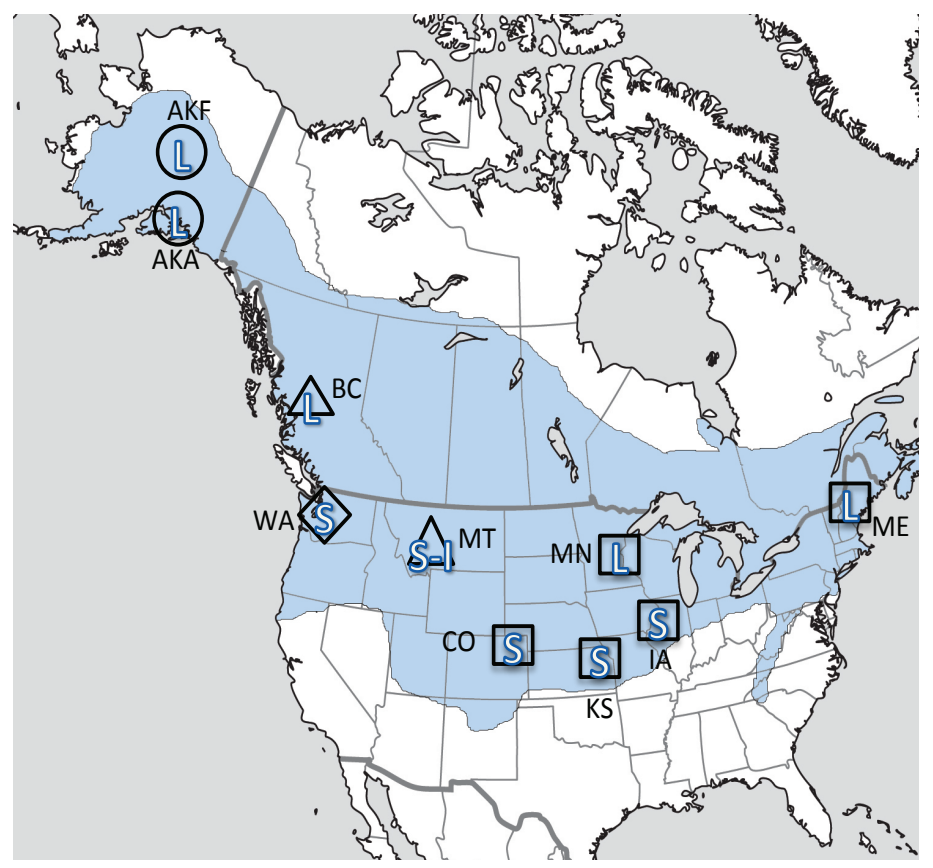


Figure 2. Hippocampus volume (A, B, D), total number of hippocampal neurons (A, B, D), and adult hippocampal neurogenesis rates (C, D) in blackcapped chickadees sampled directly from the wild without experiencing any captive environment across latitudinal (A, C) and longitudinal (B) gradient of winter climate harshness and in captive chickadees hand-reared from 10 days of age and maintained in controlled laboratory conditions throughout their entire life (D). From Roth \& Pravosudov (2009), Roth et al. (2011), and Roth et al. (2012).

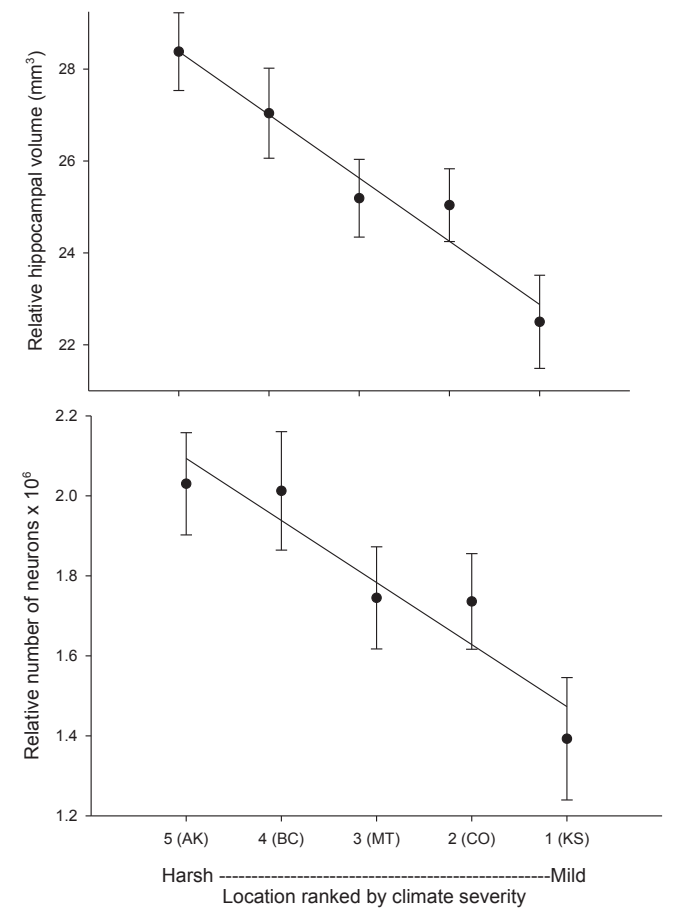

A. Black-capped chickadees: hippocampus volume and the number of neurons in wild-caught birds.
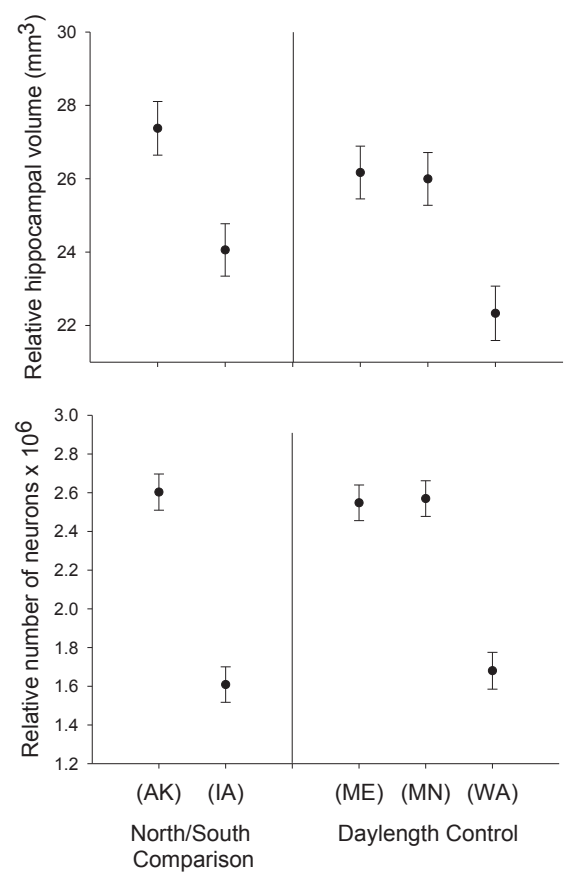

B. Black-capped chickadees: hippocampus volume and the number of neurons in wild-caught birds.

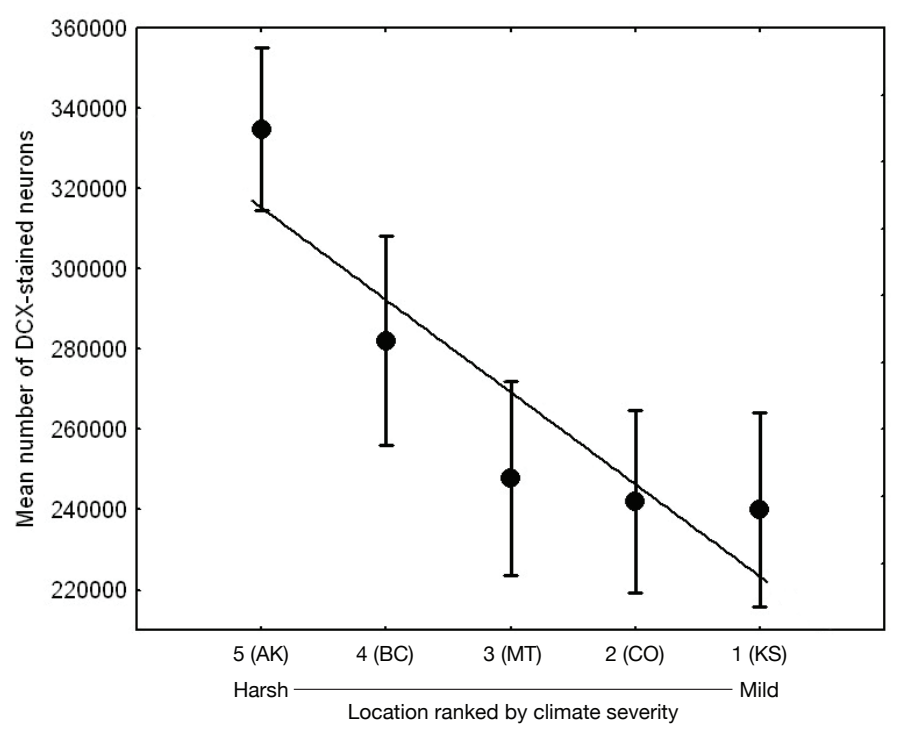

C. Black-capped chickadees: neurogenesis in wild-caught birds.

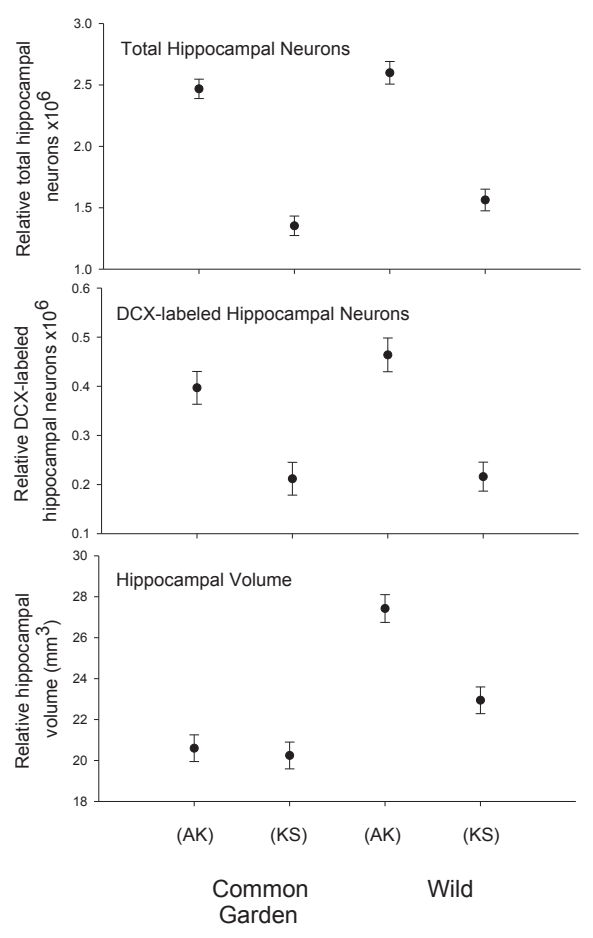

D. Black-capped chickadees: hippocampus volume, the number of neurons, and neurogenesis in hand-reared vs. wild-caught birds. 
on mountain chickadees from three different elevations are highly consistent in showing significant differences in food caching propensity, spatial memory, and hippocampus morphology related to winter climate. This pattern is, in turn, consistent with the hypothesis that population variation associated with differences in winter climate might be produced by natural selection acting on food cachingrelated spatial memory (Pravosudov \& Roth, 2013).

Harsher environments are likely associated with increased reliance on food caches for overwinter survival and therefore should favor more intense food caching and better spatial memory ability needed to recover food caches. Differential winter mortality based on individual variation in food caching propensity, spatial memory, and hippocampus morphology supporting spatial memory might be expected to result in evolutionary changes in both memory and its neural mechanisms (Pravosudov \& Roth, 2013). It is also possible that both memory and hippocampus morphology flexibly adjust to local conditions (e.g., Clayton \& Krebs, 1994; Woollett \& Maguire, 2011), and that climatedependent population variation is a product of such environment-induced phenotypic plasticity.

\section{Potential Causes of Climate-Related Variation in Spatial Memory and the Hippocampus}

Understanding the causes of climate-dependent population variation in spatial memory and the hippocampus is important for our understanding of both the evolution of cognition and how animals might respond to changing environments and to changes in climate. Most data available so far point toward natural selection acting on heritable mechanisms underlying individual differences in spatial memory and the hippocampus as the main driver for the observed climate-related variation in food-caching chickadees in the following ways:

1. Population differences in both species have been detected in juvenile birds prior to experiencing their first winter conditions even though climatic conditions during late summer and early autumn do not appear to be energetically challenging, food is usually superabundant, and chickadees mostly cache, but do not retrieve their long-term food caches (e.g., Pravosudov, 1983).

2. In both species, laboratory conditions did not eliminate population differences in food caching rates, spatial memory performance, and some hippocampal properties (most notably the total number of neurons; Freas et al., 2012; Freas, Bingman, et al., 2013; Pravosudov \& Clayton, 2002).
3. In black-capped chickadees, birds from the two extreme populations (Alaska and Kansas) were hand-reared from the nestling age when the eyes were still closed (10 days of age) and maintained in controlled laboratory conditions during their entire life. Yet hand-reared chickadees from Alaska showed higher food caching rates, displayed better spatial memory performance, were better at novel problem solving, and had significantly larger total number and soma size of hippocampal neurons, higher total number of glial cells, and higher hippocampal neurogenesis rates (Freas, Roth, et al., 2013; Roth, LaDage, Freas, \& Pravosudov, 2012). At the same time, the total number of hippocampal neurons and hippocampal neurogenesis rates were statistically similar between wild-caught and "common garden" chickadees from their respective populations. Even though the reason remains unknown, stable number of total neurons and higher neurogenesis rates in Alaska chickadees suggest higher cell death compared to more southern birds.

4. Significant differences in hippocampal gene expression were detected between "common garden" black-capped chickadees hand-reared from the two extremely different environments in genes known to be involved in neurogenesis and other hippocampal processes even though these birds spent their entire life (from day 10 of age) in the same controlled laboratory conditions (Pravosudov et al., 2013).

All of these data suggest, albeit indirectly, that population differences are unlikely to be a direct plastic response to variation in environmental conditions associated with differential demands for food caches. It remains potentially possible, however, that population differences arise following some triggers during early life or during development. If so, it appears unlikely that the nature of the potential triggers concerns some differences in food caching-related experiences. It has been shown that memory-based caching experiences are critical for hippocampus development, yet it appears that just a few caching and cache-retrieval experiences are sufficient for full hippocampus development (Clayton, 1996, 2001; Clayton \& Krebs, 1994). Considering that both black-capped and mountain chickadees cache thousands of food items starting in later summer (Brodin, 2005), it is clear that chickadees in all populations exceed the minimum threshold shown to be critical for hippocampus development (Pravosudov \& Roth, 2013). Yet, even when food caching was severely limited in laboratory conditions both in chickadees collected as juveniles after 
Figure 3. Hippocampus volume (A, D), total number of hippocampal neurons (B, E), adult hippocampal neurogenesis rates (C), and telencephalon (minus the hippocampus) volume $(F)$ in mountain chickadees sampled at different elevations directly from the wild (without experiencing captive conditions; A, B , C) and in chickadees captured as juveniles and maintained in the same controlled laboratory conditions for several months (D, E, $F-$ filled circles; open circles represent birds sampled directly from the wild for comparison). From Freas et al. (2012) and Freas, Bingman, et al. (2013).

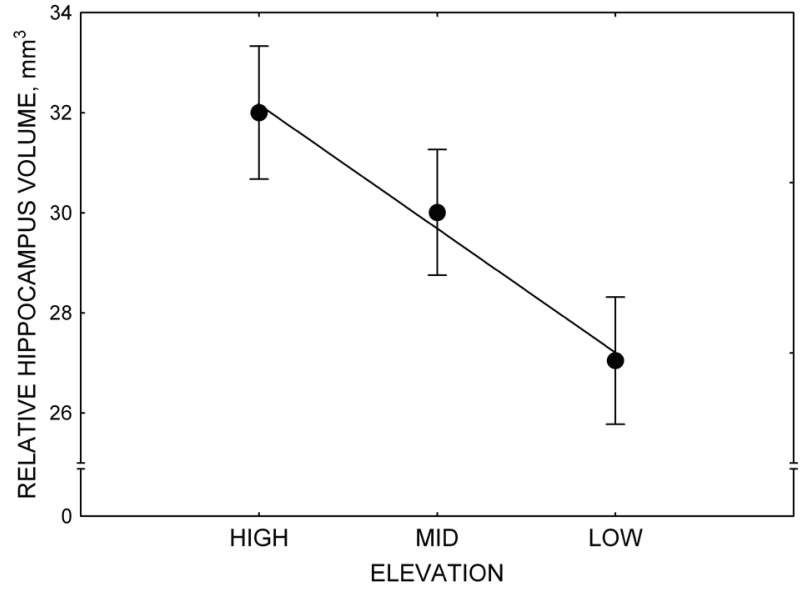

A. Mountain chickadees: hippocampus volume in wild-caught birds.

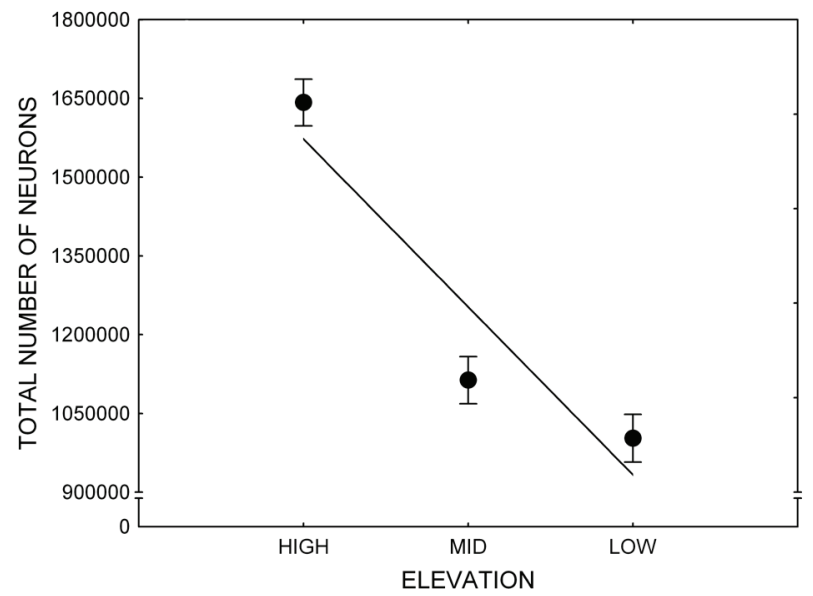

B. Mountain chickadees: the number of neurons in wild birds.

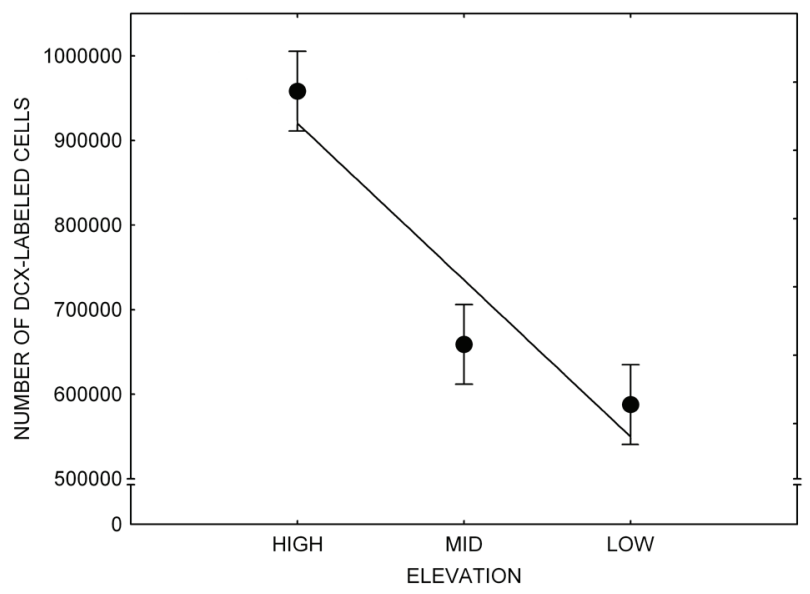

C. Mountain chickadees: neurogenesis in wild-caught birds.

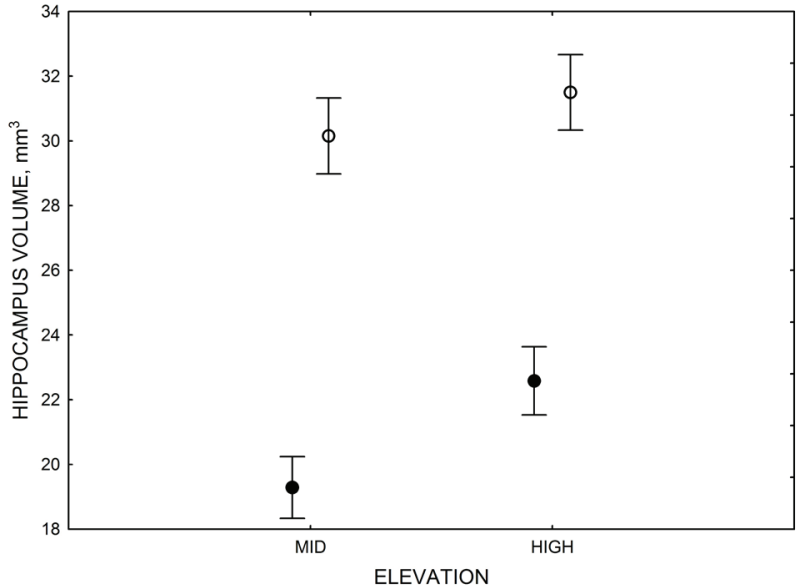

D. Mountain chickadees: hippocampus volume in captive vs. wild-caught birds.

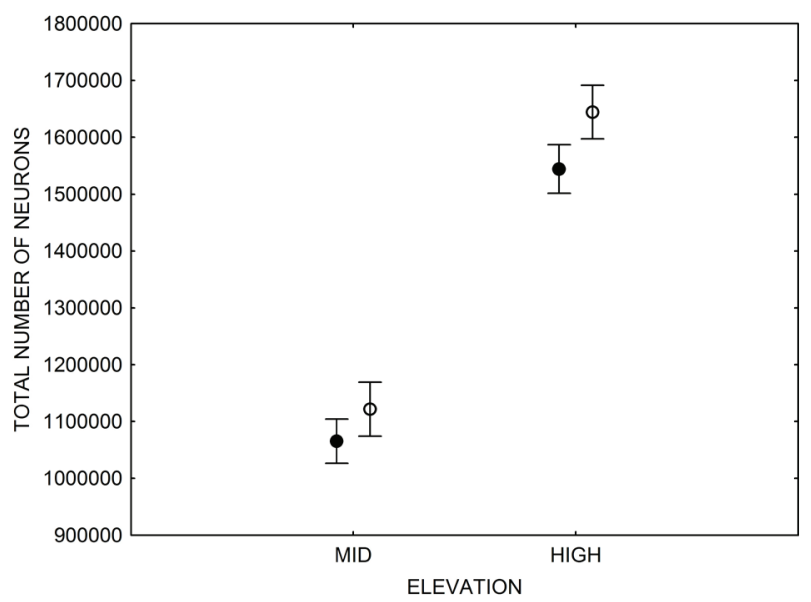

E. Mountain chickadees: the number of neurons in captive vs. wildcaught birds.

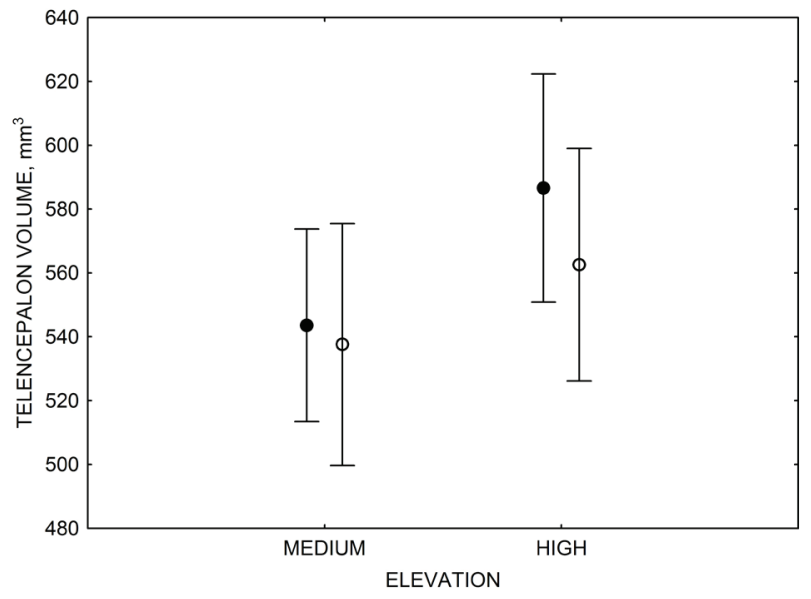

F. Mountain chickadees: telencephalon volume in captive vs. wild-caught birds. 
having some food caching experiences and in chickadees hand-reared as nestlings prior to any caching experiences, significant differences in spatial memory performance and in most hippocampal properties remained (Freas et al., 2012; Freas, Roth, et al., 2013; Roth et al., 2012). Nevertheless, the possibility that climate-related differences in memory and the hippocampus are associated with epigenetic (e.g., developmental) or maternal (e.g., yolk hormones) effects remains viable and, as of yet, untested.

\section{What Is Plastic in the Hippocampus: Experimental Studies}

Although all studies so far have been unable to eliminate population differences in memory and the hippocampus by manipulating environmental conditions, these studies provided important information about the plasticity of the hippocampus and suggested that some hippocampal properties are very plastic (e.g., hippocampus volume, neuron soma size, total number of glial cells), but others are not (total number of hippocampal neurons).

\section{Hippocampus Volume}

Many studies testing the hypothesis that interspecific variation in hippocampus size represents adaptive specialization related to memory-dependent food caching behavior (e.g., Krebs et al., 1989; Sherry et al., 1989) used hippocampus volume as a dependent measure. Population comparisons of both black-capped and mountain chickadees also used hippocampus volume among many other hippocampal properties and reported significant climate-related differences (Freas et al., 2012; Freas, Roth, et al., 2013; Pravosudov \& Clayton, 2002; Roth et al., 2011; Roth \& Pravosudov, 2009). Yet, hippocampus volume is undoubtedly one of the most plastic of all hippocampal properties. Multiple studies documented that when chickadees and other passerine birds are brought into laboratory conditions, their hippocampus volume shrinks by about 30\% (LaDage, Roth, Fox, \& Pravosudov, 2009; Smulders, Shiflett, Sperling, \& DeVoogd, 2000; Tarr, Rabinowitz, Imtiaz, \& DeVoogd, 2009). Hippocampus volume in black-capped chickadees that have been hand-reared and maintained in controlled laboratory conditions was also significantly smaller than that in chickadees sampled directly from the wild and without any period of captivity (Roth et al., 2012).

The effect of memory-based experiences on the development of the hippocampus has been well documented for young, inexperienced-in-food-caching parids (Clayton, 1996, 2001; Clayton \& Krebs, 1994). If inexperienced young birds are deprived of food caching and cache retrieval experiences, their hippocampus volume remains smaller than that of adults or young birds provided such experiences. Most important, only a few caching experiences are needed for the hippocampus to reach its full volume, and further experiences do not result in any additional increases in volume (Clayton, 2001; Clayton \& Krebs, 1994). At the same time, restriction of memory-based experiences in "experienced" birds has been suggested to result in hippocampus volume reductions (Clayton \& Krebs, 1994). This latter finding, however, was not supported by another study using wild-caught birds in a controlled laboratory environment, which showed no differences in hippocampus volume between experienced mountain chickadees deprived of food caching and cache retrieval experiences for several months and chickadees regularly engaged in these activities (LaDage et al., 2009).

It is unclear which specific mechanisms result in captivity-related changes in hippocampus volume. For birds caught as juveniles/adults and brought into captive laboratory conditions, captivity-related stress is a likely cause (Roth et al., 2012). At the same time, experimental manipulations of memory use and food caching and retrieval in captive conditions failed to produce significant differences in hippocampus volume (LaDage et al., 2009), which suggests that memory use alone might not have a strong effect on hippocampus volume in experienced birds.

It is also possible that memory use does not show any effects on hippocampus volume specifically in captive birds, which already have a much reduced hippocampus volume due to captive environment. Yet manipulations of memory use in captivity do have an effect on other hippocampal processes such as adult neurogenesis rates (LaDage, Roth, Fox, \& Pravosudov, 2010). In contrast to avian studies, human learning experiences are correlated with posterior hippocampus volume (Woollett \& Maguire, 2011), but there were no structural changes in individuals who trained, but failed to learn spatial information. It remains unclear, however, what exactly did change in the human hippocampus that resulted in an increased volume.

Seasonal changes in food caching are associated with changes in day length, yet photoperiod manipulations in captive chickadees aimed to simulate seasonal changes in day length also failed to generate significant differences in hippocampus volume, even though such manipulations affected food caching rates (Hoshooley, Phillmore, \& MacDougall-Shackleton, 2005; Krebs, Clayton, Hampton, \& Shettleworth, 1995; MacDougall-Shackleton, Sherry, Clark, Pinkus, \& Hernandez, 2003).

All in all, hippocampus volume exhibits a large degree of plasticity, but it remains unclear whether such plasticity is memory dependent in fully developed, experienced foodcaching chickadees. 


\section{Hippocampal Neuron Soma Size}

In both black-capped and mountain chickadees, hippocampal neuron soma size was significantly associated with winter climate severity, with birds in harsher environments having larger hippocampal neuron soma (Figure 4; Freas, Bingman, et al., 2013). Similar to the hippocampus volume, hippocampal neuron soma size appears highly plastic, and captivity resulted in significant soma size reduction in both black-capped and mountain chickadees (Figure 4; Freas, Bingman, et al., 2013; Freas, Roth, et al., 2013). Furthermore, it appears that captivity specifically affected neuron soma size in the hippocampus but not in the areas adjacent to the hippocampus (Freas, Bingman, et al., 2013). Despite significant reduction in hippocampal neuron soma size due to captive conditions, population differences remained significant in the hand-reared black-capped chickadees from the two extremely different environments (Freas, Bingman, et al., 2013). The fact that chickadees from the harsher environment still had significantly larger hippocampal neuron soma even though they spent their entire life (from day 10 of age) in the same controlled laboratory environment as chickadees from the milder environment suggests that these differences are regulated, at least in part, by some heritable mechanisms.

Similar to hippocampus volume, it remains unclear what exactly causes the reduction in hippocampal neuron soma size associated with a captive environment. Experimental manipulation of memory-based food caching and cache recovery did not produce any detectable effects on hippocampal neuron soma size, yet this manipulation did have a significant effect on hippocampal neurogenesis rates (Freas, Bingman, et al., 2013). So it is possible that neuron soma size reduction might be due to stress associated with captivity in birds captured as juveniles or adults (as in LaDage et al., 2009; LaDage et al., 2010). On the other hand, neuron soma were also significantly smaller in the "common garden" black-capped chickadees, which spent their entire life in controlled laboratory conditions and it is unlikely that these birds experienced captivity-associated stress similar to wildcaught birds (Freas, Bingman, et al., 2013). For example, hippocampal neurogenesis rates in these "common garden" birds were statistically indistinguishable from those in wildcaught birds that experienced natural, and unquestionably much richer, environments (Roth et al., 2012).

Overall, experimental results suggest that environmentrelated changes in hippocampus volume could be at least partially due to changes in hippocampal neuron soma size. Interestingly, captivity had no effect on telencephalon volume in chickadees (Freas, Roth, et al., 2013; LaDage et al., 2009) and also no effect on neuron soma size in telencephalic areas adjacent to the hippocampus (Freas, Bingman, et al., 2013). While it is extremely likely that captivity-associated stress is one of the drivers for such changes, it remains unclear how memory-related experiences might affect hippocampal neuron soma size. At least in captive birds collected as juveniles from the wild, manipulating the number of memory experiences failed to produce a detectable effect on hippocampal neuron soma size (Freas, Bingman, et al., 2013).

\section{Hippocampal Glia Numbers}

The total number of hippocampal glial cells was significantly different between the two populations of blackcapped chickadees from extremely different environments, with birds from harsher environment having more glia (Figure 5; Roth, LaDage, Chavalier, \& Pravosudov, 2013). At the same time, the number of glia also showed environmentinduced plasticity as chickadees that were hand-reared and maintained in the same controlled laboratory environment had significantly fewer hippocampal glia cells compared to juvenile wild-caught birds (Roth et al., 2013). Both population- and captivity-related differences in the number of hippocampal glia closely followed differences in hippocampus volume and in hippocampal neuron soma size, which suggest that plasticity in the hippocampus volume is likely due, at least in part, to changes in the number of glia. At the same time, population differences in glia still remained significant even in birds that were hand-reared and maintained in the same controlled laboratory environment-a result that suggests involvement of some heritable mechanisms underlying population differences (Roth et al., 2013). Overall, it appears that the number of hippocampal glia cells is both plastic and, to a degree, controlled by some heritable mechanisms, which might respond to selection pressure associated with environmental differences.

\section{Hippocampal Neuron Numbers}

In both black-capped and mountain chickadees, significant population differences in the total number of hippocampal neurons was associated with winter climate harshness (Freas et al., 2012; Pravosudov \& Clayton, 2002; Roth et al., 2011; Roth \& Pravosudov, 2009). Chickadees from harsher environments had significantly more hippocampal neurons. In contrast to all other, previously discussed hippocampal properties, the total number of neurons does not appear plastic. A captive environment resulted in significant reductions in hippocampus volume, neuron soma size, and glial numbers, but not in the total number of neurons (Freas, Bingman, et al., 2013; Freas, Roth, et al., 2013; LaDage et al., 2009). In mountain chickadees, two independent studies confirmed that a 
Figure 4. Mean hippocampal neuron soma size in wild black-capped chickadees (A) along environmental gradients and in wild-caught mountain chickadees (B) from different elevations. Mean hippocampal neuron soma size (C) as well as neuron soma size in brain area $\mathrm{HA}(\mathrm{G})$ and $\mathrm{M}$ mesopallium (D) in mountain chickadees from a single elevation (mid) sampled directly from the wild and captured as juveniles. These birds were maintained in laboratory conditions under two treatments: deprived (no food caching and cache retrieval experiences) and experienced (regular food caching and cache retrieval experiences). Mean hippocampal neuron soma size in black-capped chickadees (E) from two environments at the extremes of the winter harshness range sampled directly from the wild (filled circles) and hand-reared and maintained in controlled laboratory environment (open circles). Mean hippocampal neuron soma size in mountain chickadees (F) from two elevations, both sampled directly in the wild (open circles) and captured as juveniles, but maintained in a controlled laboratory environment (filled circles). From Freas, Bingman, et al. (2013).

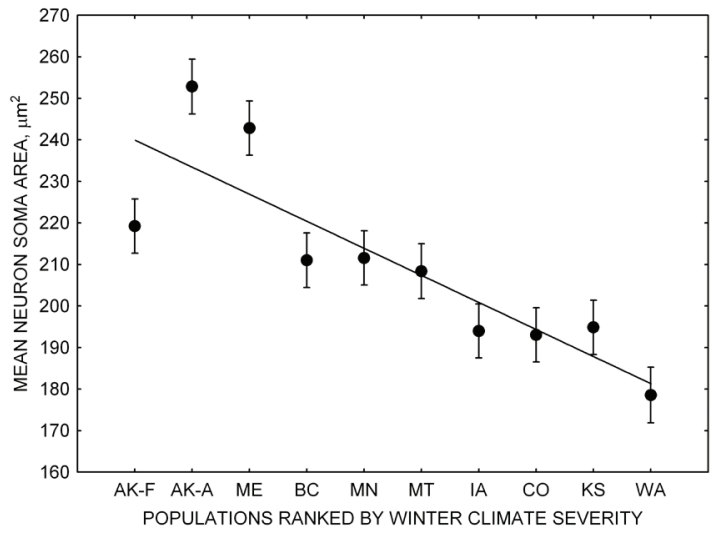

A. Black-capped chickadees: neuron soma size in wild-caught birds.

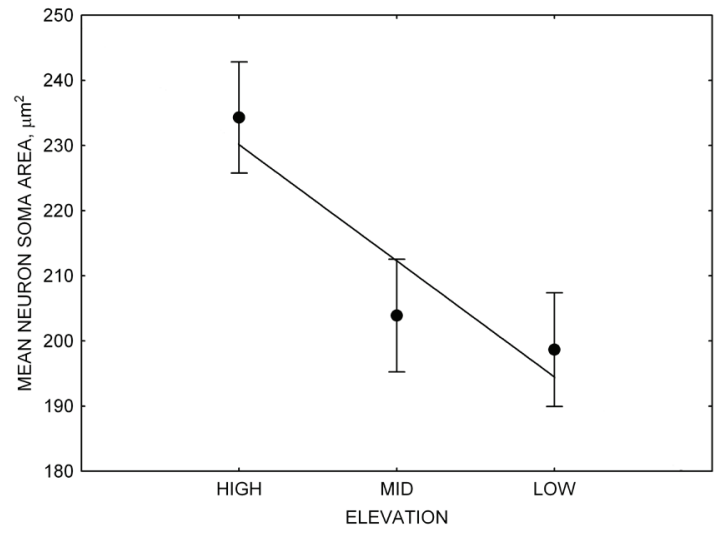

B. Mountain chickadees: neuron soma size in wild-caught birds.

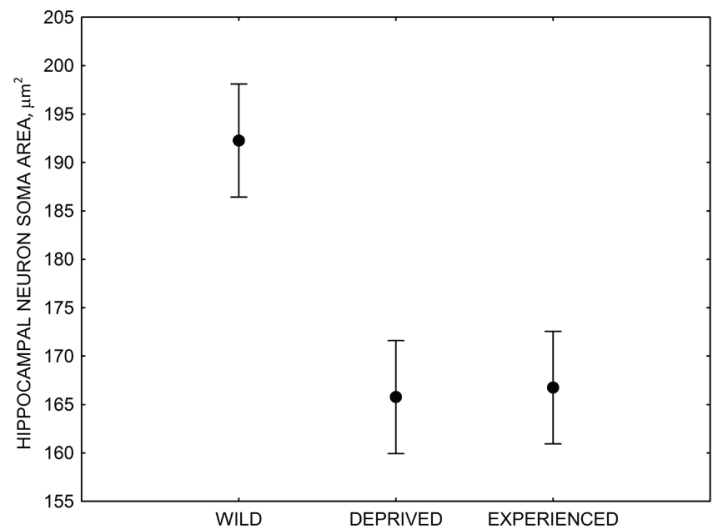

C. Mountain chickadees: hippocampal neuron soma size in wild-caught and captive birds with differences in memory use.

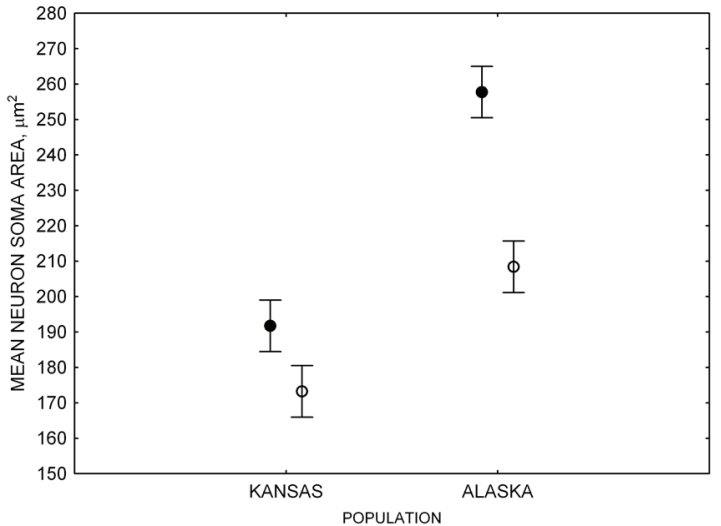

E. Black-capped chickadees: neuron soma size in hand-reared vs. wild-caught birds.

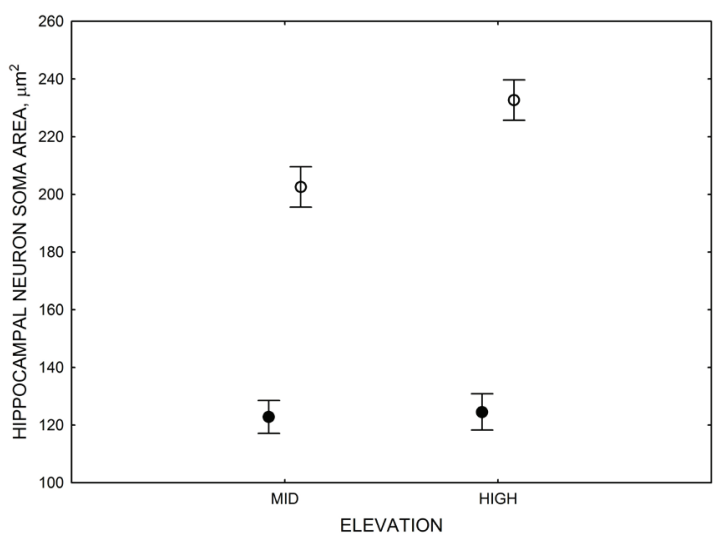

F. Mountain chickadees: neuron soma size in captive vs. wild-caught birds.

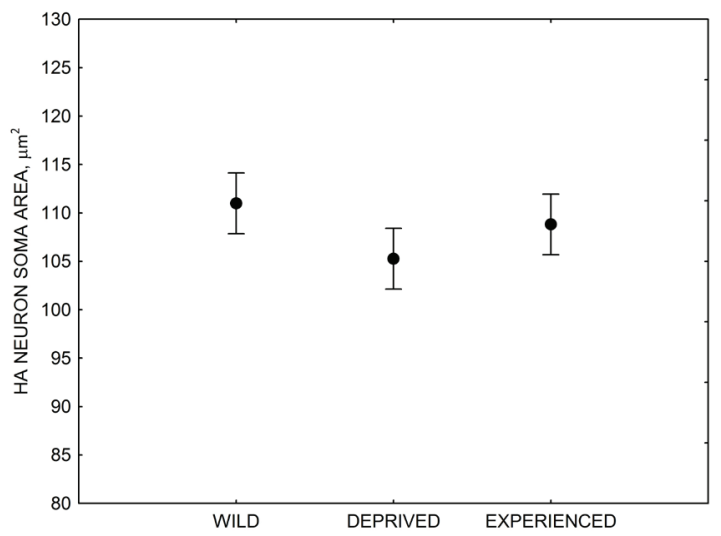

G. Mountain chickadees: HA neuron soma size in wild-caught and captive birds with differences in memory use.

(continues) 


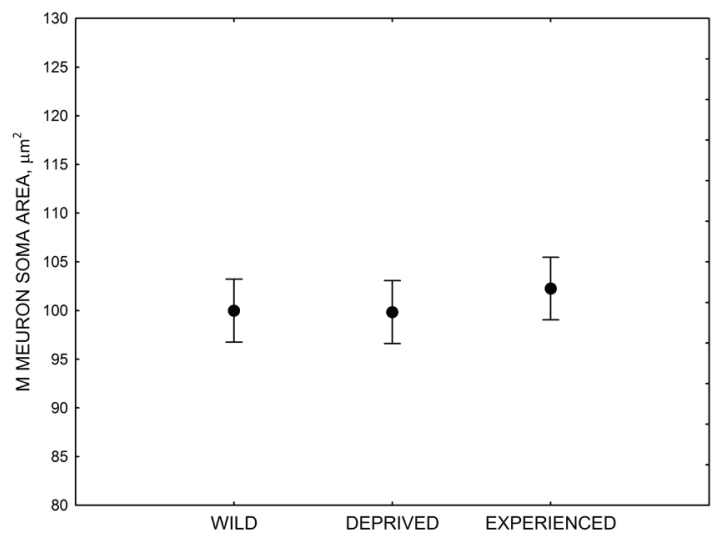

D. Mountain chickadees: M neuron soma size in wild-caught and captive birds with differences in memory use.

Figure 4 (continued). (D) in mountain chickadees from a single elevation (mid) sampled directly from the wild and captured as juveniles. These birds were maintained in laboratory conditions under two treatments: deprived (no food caching and cache retrieval experiences) and experienced (regular food caching and cache retrieval experiences). Mean hippocampal neuron soma size in black-capped chickadees

period of several months in captivity produced no significant effects on the total number of hippocampal neurons in birds collected as experienced juveniles (Figures 4, 6; Freas, Roth, et al., 2013; LaDage et al., 2009). In blackcapped chickadees, birds that were hand-reared and maintained in the same controlled laboratory environment had a statistically similar total number of hippocampal neurons to chickadees sampled as experienced juveniles in their natural environment (Figure 2; Roth et al., 2012). Furthermore, in both species, there were significant differences related to variation in winter climate in the number of hippocampal neurons both in wild-caught and captivity-maintained individuals (Freas, Roth, et al., 2013; Roth et al., 2012). Therefore, whereas population differences in hippocampus volume were associated with differences in the total number of hippocampal neurons, within-population changes in hippocampus volume were independent of the total number of neurons. These results suggest that the total number of hippocampal neurons is most likely controlled by some heritable mechanisms, which could be acted upon by natural selection. While at least some population variation in hippocampus volume might be due to potential differences in experiences, population variation in the total number of hippocampal neurons does not appear to be influenced directly by the environment. Even when hippocampus volume was reduced by as much as $30 \%$ in captivity, the number of neurons appeared to remain unchanged. So, the number of neurons might serve as

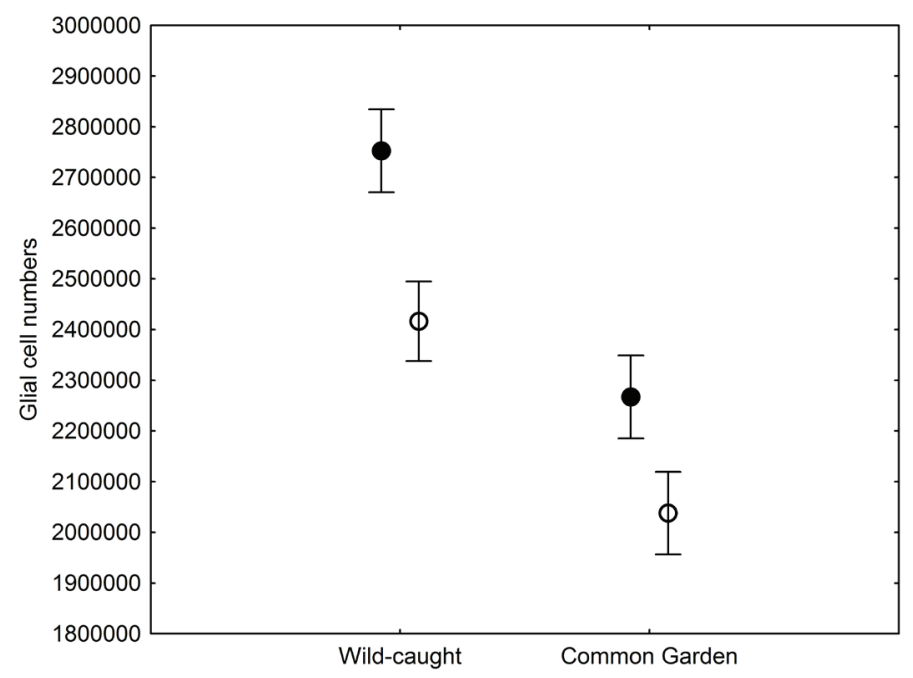

Figure 5. Mean total number of hippocampal glial cells in black-capped chickadees from two populations from the extremes of the environmental harshness range sampled both directly from the wild (filled circles) and hand-reared and maintained in the same controlled laboratory environment (open circles). From Roth et al. (2013).

a more rigid hippocampus structure, while the neuron soma size (and likely associated arborization/connectivity) and the number of glial cells are prone to changes due to immediate environmental conditions, which could produce changes in hippocampus volume independent of the number of neurons.

\section{Hippocampal Neurogenesis}

Adult hippocampal neurogenesis, a process of production, survival, and recruitment of new neurons in the hippocampus, has been generally linked to spatial learning (e.g., Barnea \& Pravosudov, 2011). As food-caching birds appear to rely on spatial memory to recover their food caches, hippocampal neurogenesis is likely an important process that might potentially be under selection. A two-species comparison indeed showed that a food-caching species had significantly higher hippocampus neurogenesis rates (Hoshooley \& Sherry, 2007). In both black-capped and mountain chickadees, adult hippocampus neurogenesis rates (estimated as the number of new immature neurons) were significantly associated with winter climate harshness, with birds from harsher climates having higher neurogenesis rates (Figures 2, 3; Chancellor et al., 2011; Freas et al., 2012). These population differences were in general agreement with the data on all other hippocampal properties: harsh winter climate was associated with larger hippocampus volume, larger total number and soma size of hippocampal neurons, larger total number of hippocampal glia cells and higher adult 
hippocampal neurogenesis rates. The question is whether these climate-related population differences in neurogenesis rates reflect plastic adjustments to local conditions and experiences or whether these differences might be, at least in part, controlled by some heritable mechanisms.

Results of experimental studies in food-caching birds suggest that adult hippocampal neurogenesis is significantly reduced in captive chickadees captured as experienced juveniles or adults (Figure 6; Barnea \& Nottebohm, 1994; LaDage et al., 2010), and that spatial memory experiences additionally affect hippocampal neurogenesis rates in wild-caught captive chickadees (LaDage et al., 2010). Mountain chickadees maintained in captive laboratory conditions, but allowed to engage in memory-based food caching and cache retrieval, had significantly higher neurogenesis rates compared to captive chickadees denied such experiences. At the same time, even experienced chickadees had significantly, and much lower, hippocampal neurogenesis rates than birds sampled directly from the wild (i.e., trapped and sacrificed without experiencing captivity; LaDage et al., 2010).

Tarr et al. (2009) was so far the only study that reported no significant effect of captivity on new hippocampal neuron survival in black-capped chickadees - a result that is strikingly different from those reported in at least two other studies (Barnea \& Nottebohm, 1994; LaDage et al., 2010). It is unclear why there was such discrepancy among the studies; in addition, Tarr et al. (2009) used methods that differ from those in all other studies. For example, Tarr et al. (2009) used multiple covariates, such as body mass, brain mass, and telencephalon volume, including the hippocampus in their analyses of the effect of captivity on the number of new cells. Use of these continuous variables as covariates can significantly affect the results concerning the effect of captivity on neuron survival rate, yet the effect of captivity on these variables has not been reported. Using the hippocampus volume as part of the overall telencephalon volume might confound the results, as the hippocampus volume is known to be affected by captivity. The question is whether new neuron survival is affected independently of any changes in the hippocampus volume. Unfortunately, Tarr et al. (2009) did not report analyses based on raw numbers of new surviving neurons, so it remains unclear whether there was an effect of captivity on the total number of new neurons. Barnea and Nottebohm (1994) reported significant reduction in new neuron survival in captive black-capped chickadees. In mountain chickadees, captivity resulted in a more than $30 \%$ reduction in the number of new immature neurons, although because of the methods used to label new neurons, this number represents a combination of new immature neurons of different age and therefore combines new neuron production and neuron survival (LaDage et al., 2010).
To add more confusion, there were no significant differences in adult neurogenesis rates (combined new neuron production and survival) between black-capped chickadees sampled directly from the wild and birds hand-reared and maintained in controlled laboratory conditions for many months (Figure 2; Roth et al., 2012). The only difference between the "common garden" study and all other chickadees studies mentioned above was that captive birds in the "common garden" study have never experienced "the wild," while in the other studies wild-caught experienced birds were brought into the lab. Such results suggest that captivity-related differences in neurogenesis rates might be directly affected by stress of captivity in wild-caught birds, whereas hand-reared birds might not be affected by such stress (Roth et al., 2012). It is also likely that most laboratory rodent and avian studies showing environmental effects on hippocampal neurogenesis (e.g., review in Barnea \& Pravosudov, 2011) also detect neurogenesis rates much below the normal "base" levels, which could indeed be improved by even slight environmental changes in extremely impoverished lab conditions. For example, Hall et al. (2014) reported significant effects of flight exercise on adult neurogenesis using doublecortin staining to quantify neurogenesis in adult starlings (Sturnus vulgaris) captured and maintained in a laboratory. The number of new neurons reported in Hall et al. (2014) is much smaller than that reported for wild chickadees using the same method (LaDage et al., 2010; Roth et al., 2012). Even though starlings are not a food-caching species and so likely have lower levels of hippocampal neurogenesis (Hoshooley \& Sherry, 2007), it is also very likely that these numbers are much reduced due to captivity and so additional exercise might simply reduce captivity-related stress's effect on neurogenesis, rather than have an additive effect on the naturally present baseline. Interestingly, photoperiod manipulations designed to imitate seasonal day length changes associated with seasonal variation in food caching activity also failed to produce any significant differences in hippocampal neurogenesis rates in captive birds (Hoshooley et al., 2005), even though such manipulations are known to affect food caching rates (MacDougall-Shackleton et al., 2003).

The major question is whether there is a threshold after which additional enhancements do not have any effects on neurogenesis. Our "common garden" experiment results certainly point in that direction as unstressed, hand-reared birds maintained in a relatively enriched captive environment (large cages, unrestricted food caching experiences) have similar hippocampal neurogenesis rates to the wild birds that experience an immensely richer natural environment. At the same time, memory experiences in likely 
Figure 6. Effect of captivity and food caching related memory use on telencephalon (minus the hippocampus) volume (A), hippocampus volume (B), total number of hippocampal neurons (C) and adult hippocampal neurogenesis rates (D, E) in mountain chickadees. From LaDage et al. (2009, 2010).

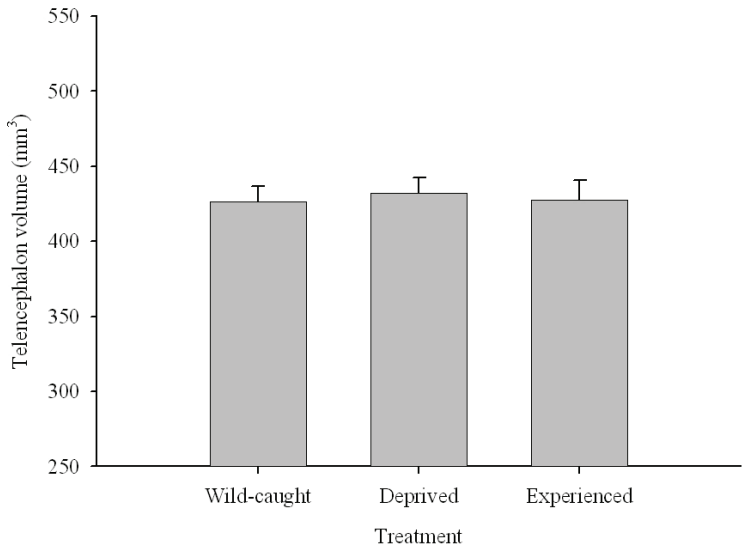

A. Mountain chickadees; telencephalon volume in wild-caught and captive birds with differences in memory use.

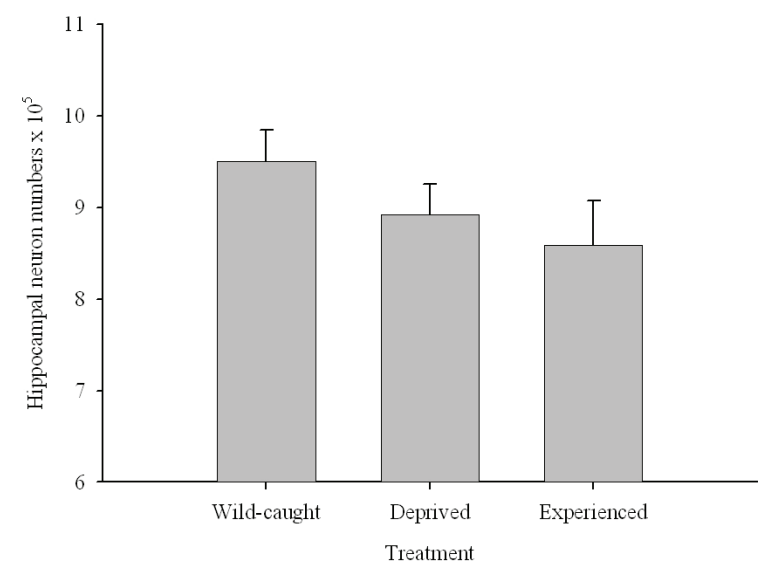

C. Mountain chickadees; hippocampal neuron numbers in wild-caught and captive birds with differences in memory use.

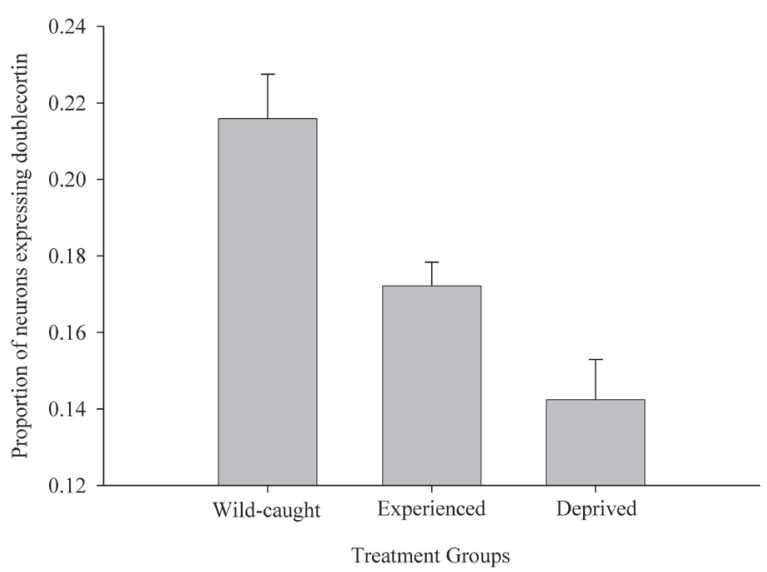

D. Mountain chickadees; proportion of new hippocampal neurons in wildcaught and captive birds with differences in memory use.

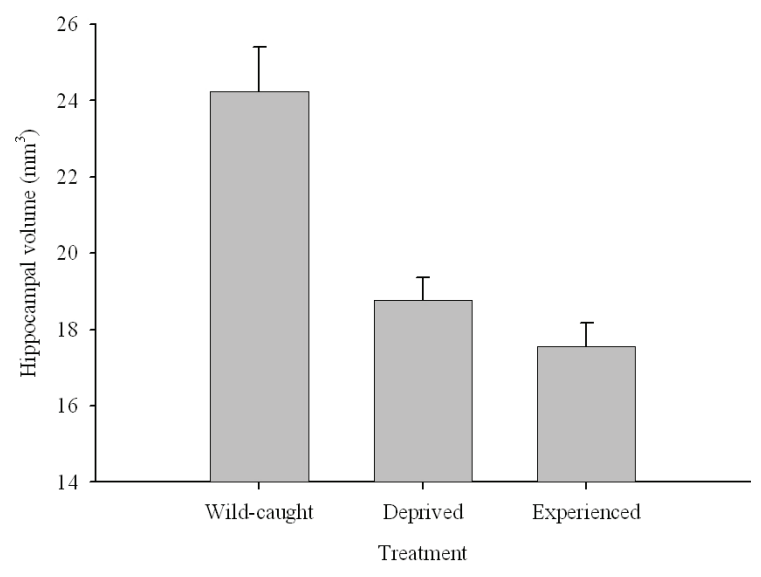

B. Mountain chickadees; hippocampus volume in wild-caught and captive birds with differences in memory use.

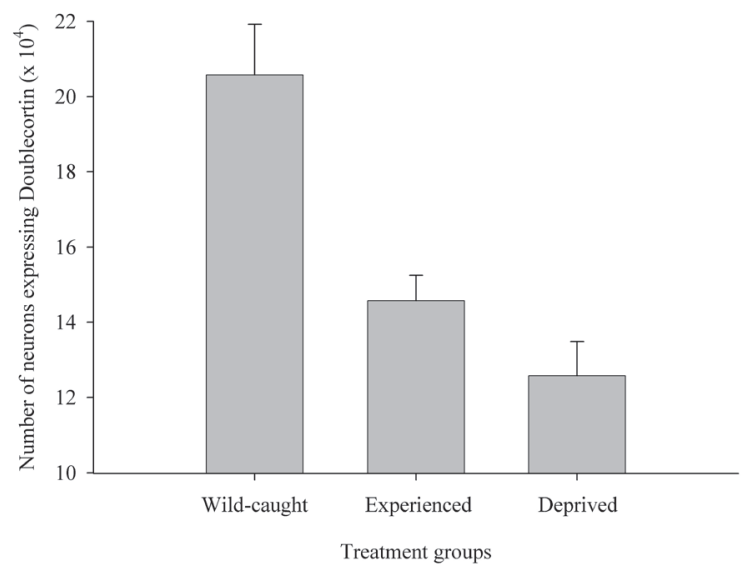

E. Mountain chickadees; hippocampal neurogenesis in wild-caught and captive birds with differences in memory use. 
stressed captive birds captured as juveniles or adults appear to ameliorate the negative effect of stress on neurogenesis (LaDage et al., 2010). Interestingly, food caching-related learning experiences have also been reported to increase hippocampal neurogenesis rates in juvenile "experiencenaïve" hand-reared marsh tits ( $P$. palustris; Patel, Clayton, $\&$ Krebs, 1997), but such an increase appears to be related to the initial memory experiences responsible for hippocampus growth and development rather than to experience-based adult neurogenesis in experienced birds.

The question remains, however, whether any additional experiences would also lead to increased neurogenesis rates given a hypothetical threshold. Results with "common garden" chickadees are certainly consistent with the threshold hypothesis as it would be difficult to explain otherwise why birds that spent their entire life in laboratory conditions had statistically indistinguishable neurogenesis rates from their conspecifics in natural conditions in the wild. These results also suggest that mechanisms regulating adult hippocampal neurogenesis rates might be heritable and therefore a potential target for natural selection acting on spatial memory.

It is also possible that food-caching species might be different from other non-caching species in maintaining hippocampal neurogenesis at high levels at all times. For example, hippocampal neurogenesis rates were almost three times as high in food-caching black-capped chickadees as in non-caching house sparrows (Passer domesticus) even after spending six weeks in captivity (Hoshooley \& Sherry, 2007).

\section{Conclusions of Experimental Studies}

Experimental studies manipulating environment/experiences in food-caching chickadees suggest that most hippocampal properties, with the exception of neuron number, are likely both plastic and at the same time controlled by some heritable mechanisms. Environment-induced plasticity in hippocampus volume appears to be related to plasticity in hippocampal neuron soma size and the number of glial cells, but not in the total number of neurons. The total number of hippocampal neurons, on the other hand, appears to be fairly constant regardless of environmental manipulations, suggesting that it is regulated by some heritable mechanism(s).

Plastic changes due to experimental manipulations in hippocampus volume, neuron soma size, and the number of glia cells also do not override population differences associated with winter climate harshness, which further suggests that such differences are likely due, at least in part, to natural selection acting on food caching-related spatial memory. It appears that the main differences among populations are based on the differences in the total number of hippocampal neurons while neuron morphology (soma) and the number of glia cells exhibit additional experience-based variation. It remains unclear, however, how much of such variation is due to differences in memory-based experiences versus stress and whether any "positive" effects in laboratory studies are still well below the baseline natural levels.

\section{Correlational Studies: Seasonal Variation}

Food-caching birds present a good case to better understand plasticity of the brain because of the highly distinct seasonality in food caching behavior (Pravosudov, 2006). Food-caching parids such as chickadees cache tens of thousands of food items during late summer-early fall (e.g., long-term caching; Brodin, 2005) and might also cache again in spring (Pravosudov, 2006), while caching much less (e.g., short-term caching) during the winter and potentially not caching at all during summer.

The three studies that brought a large amount of interest to brain plasticity associated with food caching seasonality in black-capped chickadees showed that hippocampal neuron incorporation rates were higher during late autumn (Barnea \& Nottebohm, 1994) and hippocampus volume and the total number of neurons were also highest during autumn (Smulders, Sasson, \& DeVoogd, 1995; Smulders et al., 2000). Smulders et al. (2000) used birds from the Smulders et al. (1995) study and estimated the total number of hippocampal neurons based on the hippocampus volume. These latter two studies received especially visible attention from public media, which frequently stated that foodcaching chickadees can enlarge their hippocampi by 30\% every year. Unfortunately, all available evidence combined (see below) does not support these initial claims.

First, even the initial studies provided conflicting information about seasonal changes in the number of neurons. Smulders et al. (2000) reported significant seasonal variation in the total number of hippocampal neurons, but Barnea and Nottebohm (1994) failed to detect such seasonal variation in the same species while reporting variation in hippocampal neuron incorporation rates only. At least two additional studies also failed to replicate results reported in Smulders et al. (1995) and Smulders et al. (2000) by showing no significant seasonal variation in both hippocampus volume and the total number of hippocampal neurons in black-capped chickadees (Hoshooley \& Sherry, 2004; Hoshooley, Phillmore, Sherry, \& MacDougall-Shackleton, 2007). These two latter studies also reported somewhat conflicting results on seasonal 
variation in adult hippocampal neurogenesis; Hoshooley and Sherry (2004) failed to detect significant seasonal variation in new neuron survival over 1-2 weeks, but Hoshooley et al. (2007) reported significantly higher new neuron survival rates over a 1-week period in January. Finally, Hoshooley and Sherry (2007) reported that chickadees sampled in autumn (October-November) had significantly smaller hippocampus volume and smaller number of hippocampal neurons compared to chickadees sampled in spring (March-April), a result that goes directly against the initial reports of a larger hippocampus in autumn (Smulders et al., 1995). At the same time, Hoshooley and Sherry (2007) detected no significant differences in hippocampal neurogenesis rates (new neuron survival over 6 weeks) between chickadees sampled in autumn and in spring. Finally, experimental manipulations of photoperiod in laboratory-maintained chickadees failed to produce any significant differences in hippocampus volume or hippocampal neurogenesis rates despite significantly affecting food caching rates (Hoshooley et al., 2005; Krebs at al., 1995; MacDougall-Shackleton et al., 2003). Overall, these results do not seem to provide convincing support that any of the hippocampal properties vary consistently and specifically in relation to seasonal cycle of memorybased food caching and cache retrieval. So why are there such discrepancies among the studies?

\section{Hippocampus Volume}

Using the same species in generally similar environmental conditions (Ithaca, New York and London, Ontario), one study reported significant seasonal variation in hippocampus volume (Smulders et al., 1995), the other two detected no seasonal variation (Hoshooley et al., 2007; Hoshooley \& Sherry, 2004), and the fourth actually reported that chickadees sampled in autumn had significantly smaller hippocampus volume compared to chickadees sampled in spring (Hoshooley \& Sherry, 2007). There are a couple of potential explanations for these differences.

1. Birds have been sampled in different years and in different locations, so it is possible that seasonal variation was present only in some years or only at a particular location. If that were the case, it would suggest that seasonal variation in hippocampus volume is likely not a regular phenomenon, but it might sometimes occur. Considering that winter climate conditions might be expected to be somewhat similar at both locations, this explanation does not seem likely.

2. The two labs used different methods to generate hippocampus volume estimates. Smulders et al. (1995) adjusted hippocampus volumes for the overall brain shrinkage (measured as brain mass change after post perfusion fixation process), which showed significant seasonal variation. Hoshooley and Sherry (2004, 2007) and Hoshooley et al. (2007) did not use such an adjustment. It is unfortunate that Smulders et al. (1995) did not report their data without adjusting for potential brain shrinkage so that it would be possible to evaluate whether these differences between the studies might be due to such an adjustment. At the same time, the purpose of such an adjustment is not entirely clear since hippocampus volume is measured relative to the rest of the telencephalon. In other words, even if the entire brain shrinks more, the ratio of hippocampus to telencephalon should remain the same, assuming that shrinkage is not influenced by region. Adjusting for shrinkage, on the other hand, might potentially generate spurious results specifically in regard to the relative hippocampus volume.

\section{Seasonal Variation in the Total Number of Hippocampal Neurons}

Again, seasonal variation in the total number of hippocampal neurons was reported in a single study (Smulders et al., 2000), while two other studies reported no significant seasonal variation (Barnea \& Nottebohm, 1994; Hoshooley \& Sherry, 2004) and one study actually reported the opposite pattern by showing that chickadees sampled in autumn had a significantly smaller number of hippocampal neurons than chickadees sampled in spring (Hoshooley \& Sherry, 2007). These studies did not use unbiased stereological methods (e.g., optical fractionator, West, Slomianka, \& Gunderson, 1991) to estimate the total number of neurons, but instead either counted cells only in some nonrandomly chosen areas (e.g., Smulders et al., 2000) and/or seemed to use neuron densities (number of cells divided by volume). Cell density is directly dependent on hippocampus volume and any shrinkage/variation in volume due to tissue processing could potentially produce biased results when the hippocampus volume, but not the number of neurons (or vice versa), shows significant variation. The optical fractionator method provides an estimate that is independent of tissue shrinkage or other variation in volume that is not associated with changes in neuron numbers (e.g., West et al., 1991). The optical fractionator method does depend on the volume, as a larger volume would result in more counting frames, which are used to estimate the total number of neurons. However, unlike direct density estimates (e.g., number of cells divided by volume), the optical fractionator would produce the same estimate for the number of cells if different volumes were associated with the same number of neurons. Considering that at least two studies showed no significant differences in the total number 
of hippocampal neurons between wild and captive birds using stereological methods when the hippocampus volume differed by almost 30\% (Freas, Roth, et al., 2013; LaDage et al., 2009), it does not seem likely that chickadees would exhibit regular significant seasonal variation in the total number of hippocampal neurons. In fact, black-capped chickadees sampled at almost the same time when Smulders et al. (2000) reported a significant peak in the number of neurons (October) had a statistically indistinguishable number of hippocampal neurons from those in chickadees that were hand-reared and maintained in controlled laboratory conditions and were sampled in spring (Roth et al., 2012). If the number of neurons reflected differences in memory-based food caching, it should be expected that wild chickadees at the peak of food caching should experience much higher memory demands than hand-reared birds living in relatively small cages, yet these two groups did not differ significantly in the total number of neurons (Roth et al., 2012). Finally, Hoshooley and Sherry (2007) also reported a higher number of hippocampal neurons in spring compared to autumn - a pattern opposite to the one suggested by Smulders et al. (2000).

While it is impossible to say why only one of the four studies was able to report seasonal differences in the number of hippocampal neurons, considering all correlational and experimental evidence, it does not appear likely that the number of hippocampal neurons regularly exhibits food caching-related seasonal variation.

\section{Hippocampal Neurogenesis}

Data on seasonal variation in hippocampal neurogenesis rates in food-caching chickadees is also quite inconsistent. First, Barnea and Nottebohm (1994) reported that hippocampal new neuron incorporation rates were highest in black-capped chickadees injected with new neuron marker in October and attributed these high rates to the peak of autumn food caching. Hoshooley and Sherry (2004, 2007) reported no significant seasonal variation in hippocampal neurogenesis rates in the same species, and Hoshooley et al. (2007) reported a peak in new hippocampal neuron survival rates in January (and potentially in April when neurogenesis rates were not statistically different from those sampled in January), much later than reported by Barnea and Nottebohm (1994).

Hippocampal neurogenesis is the only hippocampal attribute (among the ones considered here) that has indeed been experimentally linked to spatial memory use (LaDage et al., 2010). Based on such experimental evidence it might be plausible to expect that seasonal changes in memory use associated with food caching might indeed produce seasonal changes in hippocampal neurogenesis rates. Yet available evidence does not seem to provide unequivocal support for the idea that changes in hippocampal neurogenesis rates track seasonal changes in memory use associated with food caching.

It is likely that chickadees use spatial memory both when they make tens of thousands of food caches during later summer-early fall (e.g., Male \& Smulders, 2007) as well as all throughout the winter when they recover these caches (see references in Pravosudov \& Smulders, 2010). So it is not clear whether memory use (all aspects, including memory acquisition during caching, memory formation, and memory recall used either during cache retrieval or when making other caches relative to locations of previously made caches) should be higher during the peak of caching or the entire winter. See Barnea and Pravosudov (2011) for more discussion about neurogenesis.

If memory use is heaviest during the peak of caching, it might be expected that the highest neurogenesis rates should be in late August-September and early October at the latest (Pravosudov, 2006). If new neurons are needed for new memories, new neurons should be incorporated into the existing hippocampal circuits during that time and new neuron production could be triggered at the beginning of intense food caching in late August. Yet, Barnea and Nottebohm (1994) detected highest new neuron incorporation rates 6 weeks after injecting birds with a new neuron marker in October. So these new neurons were likely functional only in mid to late November, much later and after the peak of food caching and therefore unlikely related to memory needs associated with food caching (e.g., Barnea \& Pravosudov, 2011). Results of Hoshooley and Sherry (2007) showed an even later peak in new neuron survival (January), which is not likely related to the food caching process.

If memory use is the highest during cache retrieval, it might be expected that food-caching chickadees use memory intensely during the entire winter, or at least during a few winter months, likely from November to February. The data from both Barnea and Nottebohm (1994) and Hoshooley et al. (2007) still do not fit such a pattern. Barnea and Nottebohm (1994) reported the highest neuron incorporation rates only in birds injected with new neuron marker in October (measured 6 weeks later-likely in late November), but not in birds injected in December even though cache retrieval memory use should be as high in January as in November. Hoshooley et al. (2007), on the other hand, reported the highest hippocampal neuron 1-week survival rates in birds sampled in January-February, yet new neuron survival rates were almost as high (and statistically indistinguishable from) new neuron survival rates in birds sampled in April-May, when cache retrieval should not be critical. 
At the same time, Hoshooley and Sherry $(2004,2007)$ did not detect any significant seasonal variation in hippocampal neurogenesis rates.

There are important differences between the Barnea and Nottebohm (1994), the Hoshooley and Sherry (2004), and the Hoshooley et al. (2007) studies concerning the measured period of new neuron survival (Barnea \& Pravosudov, 2011). While Barnea and Nottebohm (1994) and Hoshooley and Sherry (2007) estimated 6-week survival, Hoshooley and Sherry (2004) and Hoshooley et al. (2007) measured 1-2 week survival. In the latter two studies and in Hoshooley and Sherry (2007), neuron survival was measured in captive birds, while Barnea and Nottebohm (1994) measured neuronal incorporation rates in free-ranging birds. Despite these differences, the observed patterns do not seem to fit any of the patterns predicted using seasonality of food caching and cache retrieval. One-to-two week survival might be potentially insufficient to detect important differences in neuron survival, as it may take more than 6 weeks for the new neurons to express adult phenotype (Hoshooley \& Sherry, 2007), so the data presented in Hoshooley and Sherry (2004) and Hoshooley et al. (2007) might be more indicative of new neuron production rates. Yet, seasonal variation in 6-week survival rates reported in Barnea and Nottebohm (1994) still does not follow a pattern expected from seasonal variation in food caching and cache retrieval.

Finally, there are methodological differences concerning using tritiated thymidine (Barnea \& Nottebohm, 1994) and BrdU (Hoshooley \& Sherry's studies) that might also produce potential differences in estimation of neurogenesis rates (Leuner, Glasper, \& Gould, 2009).

Overall, the available data do not seem to provide clear evidence for robust food caching-related seasonal variation in adult hippocampal neurogenesis rates. While it is possible that there are some seasonal changes, they might be unrelated to food caching and associated with some other factors such as winter temperature or activity patterns. While chickadees captured as juveniles and maintained in captive conditions did show memory use-based increases in hippocampal neurogenesis, these increases did not compensate for the large captivity-related reduction in neurogenesis rates (LaDage et al., 2010). At the same time, black-capped chickadees hand-reared and maintained in laboratory conditions had statistically similar hippocampal neurogenesis rates (joint estimate of new neuron production and survival) to those in chickadees sampled directly from the wild during the peak of food caching (Roth et al., 2012). There is little doubt that birds in the wild must have more memory-based experiences than birds that spent their entire life in a relatively confined captive environment, yet such differences were not reflected by hippocampal neurogenesis rates. Such data are suggestive of some rather small threshold beyond which more experiences are not likely to produce an additional increase in hippocampal neurogenesis. Such a suggestion, however, remains a speculation at this point, and more data are needed to understand the patterns of association between memory use and neurogenesis.

Overall, there appears to be no clear evidence that the hippocampus undergoes robust and predictable seasonal changes associated specifically with food caching and/or cache retrieval. In fact, many studies reported no significant seasonal variation in any of the traits-hippocampus volume, total neuron numbers, or adult neurogenesis rates.

\section{Overall Conclusions}

Population comparisons of two species of food-caching chickadees experiencing different winter climate conditions provided highly consistent evidence of environment-related, strong variation in spatial memory, hippocampus morphology including hippocampus volume, total number and soma size of hippocampal neurons, total number of hippocampal glia, and adult hippocampal neurogenesis rates.

Experimental data suggest that some, but not all, of these hippocampal properties might be directly affected by the environment; however, in all cases the largest effects were due to captive environment. Memory-based experiences were only shown to up-regulate hippocampal neurogenesis rates in captive birds with neurogenesis rates already significantly reduced in captive conditions. All other hippocampal properties discussed here were unaffected by manipulations of such experiences. In contrast, birds that were hand-reared from an early age and maintained in a fairly enriched laboratory environment (large cages, ability to cache food in multiple substrates) had adult hippocampal neurogenesis rates statistically indistinguishable from those measured in wild birds in their immensely richer natural environment, which points toward a relatively small threshold in experiences beyond which adult neurogenesis rates do not appear to be affected by additional enriching experiences.

The fact that hippocampus volume might be affected by the environment without significant changes in the total number of neurons suggests that using neuron densities for evaluating cognitive abilities is not only incorrect, but could be misleading. For example, captivity is associated with a significant reduction in hippocampus volume, but not in the number of neurons, which results in higher density of hippocampal neurons in captive birds.

Most evidence is consistent with the hypothesis that climate-related population variation in spatial memory and 
hippocampus morphology is produced by natural selection associated with individual heritable variation in spatial memory and its neural mechanisms. The fact that the total number of neurons does not change, even in extremely impoverished captive conditions, suggests the involvement of some heritable regulatory mechanisms. While the hippocampus volume, total number of glia, and neuron soma size can and do respond to direct environmental changes, these changes appear to be anchored around the total number of neurons, which seems quite stable. Although it remains untested whether individual variation in spatial memory and hippocampal morphology in birds is heritable and based on genetic variation, there is evidence from human research showing heritability of general cognitive ability, spatial ability, and hippocampus volume, as well as its genetic basis (e.g., Ando et al., 2001; Haworth et al., 2010; McGee, 1979; Pedersen et al., 1992; Plomin et al., 1994; Plomin \& Spinath, 2002; Sullivan, Pfefferbaum, Swan, \& Carmelli, 2001). Finally, there appears to be no unambiguous evidence showing consistent seasonal variation in hippocampus morphology directly related to the seasonal cycle of food caching and cache retrieval. In fact, experimental data on the number of neurons suggests that at least the number of neurons is not likely to vary seasonally.

Overall, it appears that environment-induced plasticity in hippocampus morphology related to hippocampus volume, total number and size of hippocampal neurons, glia cell numbers, and even hippocampal neurogenesis rates might be anchored around the total number of hippocampal neurons, which appears to be regulated by some heritable mechanisms responsive to natural selection on food caching-related spatial memory. More research on hippocampus plasticity needs to be done on wild birds as captive conditions generate strong negative effects and all experience-based experimental manipulations in captive birds, especially captured as juvenile or adults, cannot come close to the baseline levels present in wild birds. Such strong captivity effects suggest that any results of experimental studies investigating brain plasticity should be considered cautiously.

\section{References}

Ando, J., Ono, Y., \& Wright, M. J. (2001). Genetic structure of spatial and verbal working memory. Behavior Genetics, 31, 615-624. doi:10.1023/A:1013353613591
Barnea, A., \& Nottebohm, F. (1994). Seasonal recruitment of hippocampal neurons in adult free-ranging blackcapped chickadees. Proceedings of the National Academy of Sciences of the United States of America, 91, 11271-11221.

Barnea, A., \& Pravosudov, V. V. (2011). Birds as a model to study adult neurogenesis: Bridging evolutionary, comparative and neuroethological approaches. European Journal of Neuroscience, 34, 884-907. doi:10.1111/j.1460-9568.2011.07851.x

Brodin, A. (2005). Hippocampal volume does not correlate with food-hoarding rates in the blackcapped chickadee (Poecile atricapillus) and willow tits (Parus montanus). Auk, 122, 819-828. doi:10.1642/0004-8038(2005)122[0819:HVDNCW]2 .0. $\mathrm{CO} ; 2$

Chancellor, L. V., Roth, T. C., II, LaDage, L. D., \& Pravosudov, V. V. (2011). The effect of environmental harshness on neurogenesis: A large-scale comparison. Developmental Neurobiology, 71, 246-252. doi:10.1002/dneu.20847

Clayton, N. S. (1996). Development of food-storing and the hippocampus in juvenile marsh tits (Parus palustris). Behavioral Brain Research, 74, 153-159. doi:10.1016/0166-4328(95)00049-6

Clayton, N. S. (2001). Hippocampal growth and maintenance depend on food-caching experience in juvenile mountain chickadees (Poecile gambeli). Behavioral Neuroscience, 115, 614-625. doi:10.1037/0735-7044.115.3.614

Clayton, N. S., \& Krebs, J. R. (1994). Hippocampal growth and attrition in birds affected by experience. Proceedings of the National Academy of Sciences of the United States of America, 91, 7410-7414. doi:10.1073/pnas.91.16.7410

Ekman, J. (1989). Ecology of non-breeding social systems of Parus. Wilson Bulletin, 101, 263-288.

Freas, C., LaDage, L. D., Roth, T. C., II, \& Pravosudov, V. V. (2012). Elevation-related differences in memory and the hippocampus in food-caching mountain chickadees. Animal Behaviour, 84, 121-127. doi:10.1016/j.anbehav.2012.04.018 
Freas, C. A., Bingman, K., LaDage, L. D., \& Pravosudov, V. V. (2013). Untangling elevation-related differences in the hippocampus in food-caching mountain chickadees: The effect of a uniform captive environment. Brain, Behavior and Evolution, 82, 199-209. doi:10.1159/000355503

Hall, Z. J., Bauchinger, U., Gerson, A. R., Price, E. R., Langlois, L. A., Boyles, M., et al. (2014). Site-specific regulation of adult neurogenesis by dietary fatty acid content, vitamin E and flight exercise in European starlings. European Journal of Neuroscience, 39, 875-882. doi:10.1111/ejn.12456

Haworth, C. M. A., Wright, M. J., Luciano, M., Martin, N. G., de Geus, E. J. C., van Beijsterveldt, C. E. M., et al. (2010). The heritability of general cognitive ability increases linearly from childhood to young adulthood. Molecular Psychiatry, 15, 1112-1120. doi:10.1038/mp.2009.55

Hogstad, O. (1989). Social organization and dominance behavior in some Parus species. Wilson Bulletin, 101, 254-262.

Hoshooley, J. S., Phillmore, L. S., \& MacDougallShackleton, S. A. (2005). An examination of avian hippocampal neurogenesis in relationship to photoperiod. Neuroreport, 16, 987-991. doi:10.1097/00001756-200506210-00021

Hoshooley, J. S., Phillmore, L. S., Sherry, D. F., \& MacDougall-Shackleton, S. A. (2007). Annual cycle of the black-capped chickadee: Seasonality of foodstoring and the hippocampus. Brain Behavior and Evolution, 69, 161-168. doi:10.1159/000096984

Hoshooley, J. S., \& Sherry, D. F. (2004). Neuron production, neuron number, and structure size are seasonally stable in the hippocampus of the food-storing black-capped chickadee (Poecile atricapillus). Behavioral Neuroscience, 118, 345-355. doi:10.1037/0735-7044.118.2.345

Hoshooley, J. S., \& Sherry, D. F. (2007). Greater hippocampal neuronal recruitment in food-storing than in non-food-storing species. Developmental Neurobiology, 67, 406-414. doi:10.1002/dneu.20316
Krebs, J. R., Clayton, N. S., Hampton, R. R., Shettleworth, S. J. (1995). Effects of photoperiod on food-storing and the hippocampus in birds. Neuroreport, 6 , 1701-1704. doi:10.1097/00001756-199508000-00026

Krebs, J. R., Sherry, D. F., Healy, S. D., Perry, V. H., \& Vaccarino, A. L. (1989). Hippocampal specialization of food-storing birds. Proceedings of the National Academy of Sciences of the United States of America, 86, 1388-1392. doi:10.1073/pnas.86.4.1388

LaDage, L. D., Roth, T. C., II, Fox, R. A., \& Pravosudov, V. V. (2009). Effects of captivity and memorybased experiences on the hippocampus in mountain chickadees. Behavioral Neuroscience, 123, 284-291. doi:10.1037/a0014817

LaDage, L. D., Roth, T. C., II, Fox, R. A., \& Pravosudov, V. V. (2010). Ecologically-relevant spatial memory use modulates hippocampal neurogenesis. Proceedings of the Royal Society B: Biological Sciences, 277, 10711079. doi:10.1098/rspb.2009.1769

Leuner, B., Glasper, E. R., \& Gould, E. (2009). Thymidine analog methods for studies of adult neurogenesis are not equally sensitive. Journal of Comparative Neurology, 517, 123-133. doi:10.1002/cne.22107

Male, L. H., \& Smulders, T. V. (2007). Memory for food caches: Not just for retrieval. Behavioral Ecology, 18, 456-459. doi:10.1093/beheco/arl107

MacDougall-Shackleton, S. A., Sherry, D. F., Clark, A. P., Pinkus, R., \& Hernandez, A. M. (2003). Photoperiodic regulation of food storing and hippocampus volume in black-capped chickadees, Poecile atricapillus. Animal Behavior, 65, 805-812. doi:10.1006/anbe.2003.2113

McGee, M. G. (1979). Human spatial abilities: Psychometric studies and environmental, genetic, hormonal, and neurological influences. Psychological Bulletin, 86, 889-918. doi:10.1037/0033-2909.86.5.889

Patel, S. N., Clayton, N. S., \& Krebs, J. R. (1997). Spatial learning induces neurogenesis in the avian brain.

Behavioral Brain Research, 89, 115-128. doi:10.1016/S0166-4328(97)00051-X 
Pedersen, N. L., Plomin, R., Nesselroade, J. R., \& McClearn, G. E. (1992). A quantitative genetic analysis of cognitive abilities during the second half of the life span. Psychological Science, 3, 346-353. doi:10.1111/j.1467-9280.1992.tb00045.x

Plomin, R., \& Spinath, F. M. (2002). Genetics and general cognitive ability (g). Trends in Cognitive Sciences, 6 , 169-176. doi:10.1016/S1364-6613(00)01853-2

Pravosudov, V. V. 1985. Search for and storage of food by Parus cinctus lapponicus and P. montanus borealis (Paridae). Zoologichesky Zhurnal, 64(7): 1036-1043.

Pravosudov, V. V. (2006). On seasonality of food caching behavior in parids: Do we know the whole story? Animal Behaviour, 71, 1455-1460. doi:10.1016/j.anbehav.2006.01.006

Pravosudov, V. V., \& Clayton, N. S. (2002). A test of the adaptive specialization hypothesis: Population differences in caching, memory and the hippocampus in black-capped chickadees (Poecile atricapilla). Behavioral Neuroscience, 116, 515-522. doi:10.1037/0735-7044.116.4.515

Pravosudov, V. V., \& Roth, T. C., II. (2013). Cognitive ecology of food hoarding: The evolution of spatial memory and the hippocampus. Annual Reviews of Ecology, Evolution and Systematics, 44, 18.1-18.21. doi:10.1146/annurev-ecolsys-110512-135904

Pravosudov, V. V., Roth, T. C., II, Forister, M., LaDage, L. D., Kramer, R., Schilkey, F., et al. (2013). Differential hippocampal gene expression associated with climate-related natural variation in memory and the hippocampus in food-caching chickadees. Molecular Ecology, 22, 397-408. doi:10.1111/mec.12146

Pravosudov, V. V., \& Smulders, T. V. (2010). Integrating ecology, psychology, and neurobiology within a foodhoarding paradigm, Philosophical Transactions of the Royal Society B: Biological Sciences, 365, 859-867. doi:10.1098/rstb.2009.0216

Roth II, T. C., LaDage, L. D., Chavalier, D., \& Pravosudov, V. V. (2013). Variation in hippocampal glial cell numbers in food-caching birds from different climates. Developmental Neurobiology, 73, 480-485. doi:10.1002/dneu.22074
Roth, T. C., II, LaDage, L. D., \& Pravosudov, V. V. (2011). Variation in hippocampal morphology along an environmental gradient: Controlling for the effect of day length. Proceedings of the Royal Society B: Biological Sciences, 278, 2662-2667. doi:10.1098/ rspb.2010.2585

Sherry, D. F., Vaccarino, A. L., Buckenham, K., \& Herz, R. S. (1989). The hippocampal complex of food-storing birds. Brain Behavior and Evolution, 34, 308-317. doi:10.1159/000116516

Smulders, T. V., Sasson, A. D., \& DeVoogd, T. J. (1995). Seasonal variation in hippocampal volume in a foodstoring bird, the black-capped chickadee. Journal of Neurobiology, 27, 15-25. doi:10.1002/neu.480270103

Smulders, T. V., Shiflett, M. W., Sperling, A. J., \& DeVoogd, T. J. (2000). Seasonal changes in neuron numbers in the hippocampal formation of a foodhoarding bird: The black-capped chickadee. Journal of Neurobiology, 44, 414-422. doi:10.1002/1097 -4695(20000915)44:4<414::AID-NEU4>3.0.CO;2-I

Sullivan, E. V., Pfefferbaum, A., Swan, G. E., \& Carmelli, D. (2001). Heritability of hippocampal size in elderly men: Equivalent influence from genes and environment. Hippocampus, 11, 754-762. doi:10.1002/hipo.1091

Tarr, B. A., Rabinowitz, J. S., Imtiaz, M. A., \& DeVoogd, T. J. (2009). Captivity reduces hippocampal volume but not survival of new cells in a food-storing bird. Developmental Neurobiology, 69, 972-981. doi:10.1002/dneu.20736

Vander Wall, S. B. (1990). Food hoarding in animals. University of Chicago Press.

West, M. J., Slomianka, L., \& Gunderson, H. J. (1991). Unbiased stereological estimation of the total number of neurons in the subdivisions of the rat hippocampus using the optical fractionator. Anatomical Record, 231, 482-497. doi:10.1002/ar.1092310411

Woollett, K., \& Maguire, E. A. (2011). Acquiring "the Knowledge" of London's layout drives structural brain changes. Current Biology, 21, 2109-2114. doi:10.1016/j.cub.2011.11.018 